
distributed under the terms of the Creative Commons Attribution licence (http://creativecommons.org/licenses/by/4.0/), which permits unrestricted re-use, distribution, and reproduction in any medium, provided the original work is properly cited.

\title{
Hot Politics? Affective Responses to Political Rhetoric
}

\author{
BERT N. BAKKER University of Amsterdam \\ GIJS SCHUMACHER University of Amsterdam \\ MATTHIJS ROODUIJN University of Amsterdam
}

\begin{abstract}
$C$ anonical theories of opinion formation attribute an important role to affect. But how and for whom affect matters is theoretically underdeveloped. We establish the circumplex model in political science as a theory of core affect. In this theory unconscious emotional processes vary in level (arousal, measured with skin conductance) and direction (valence, measured with facial electromyography). We theorize that knowledge, attitude extremity, and (in) congruence with political rhetoric explain variation in affective responses. In a large lab study $(\mathrm{N}=397)$, participants watched video clips with left-wing or rightwing rhetoric on prominent issues. We find that people with extreme attitudes experience more arousal in response to political rhetoric and that political rhetoric incongruent with prior attitudes evokes negative affect. Moreover, we show that affective responses lead to opinion change, independent of self-reported emotions. We conclude by setting a research agenda for the alignment between affective and cognitive components of emotions and their consequences.
\end{abstract}

$\mathbf{S}$ ome people say they vote with their gut. Other people say that they follow their heart. These metaphors are meant to reveal the affective nature of political decision making. This affective nature is also central to key political decision-making models such as motivated reasoning (Lodge and Taber 2013), affective intelligence (Marcus, Neuman, and MacKeun 2000), receive-accept-sample (Zaller 1992), and directional voting (Rabinowitz and Macdonald 1989). These very different models link affect-or allied terms such as feelings or emotions - to biases in information processing, which in turn motivate different attitudinal and/or behavioral outcomes. However, affect remains underdeveloped in these models. We observe that

Bert N. Bakker (D), Assistant Professor, Amsterdam School of Communication Research, University of Amsterdam, b.n.bakker@uva.nl.

Gijs Schumacher (D), Associate Professor, Department of Political Science, University of Amsterdam, g.schumacher@uva.nl.

Matthijs Rooduijn (iD, Assistant Professor, Department of Political Science, University of Amsterdam,m.rooduijn@uva.nl.

Support for this research was provided by the Dutch National Science Foundation (B.N.B., G.S. \& M.R.), the Royal Dutch Academy of Sciences (B.N.B., G.S., \& M.R.), the broadcaster NTR (B.N.B., G.S., \& M.R.), BKB: The Campaign Agency (B.N.B., G.S., \& M.R.), the University of Amsterdam (B.N.B., G.S., \& M.R.), the Amsterdam School of Communication Research (B.N.B.), the Research Priority Area Communication (B.N.B.), the European Union's Horizon 2020 research and innovation programme under grant agreement No. 750443 (B.N.B), the European Research Council (ERC) under the European Union's Horizon 2020 research and innovation programme under grant agreement No 759079 (G.S), and an NWO-Veni grant No 451-16-020 (M.R). We thank Claes de Vreese for his support of this project when it was just an idea. We want to thank the organizers of "Lowlands Science" and in particular Noortje Jakobs, Rewan Janssen, and the late Erik van Bruggen for the facilitation of the data collection in protocol 1. Gé Teunissen, Sjoukje Kerman, and Robin Meijers facilitated the creation of the stimulus material of protocol 1. We want to thank the team of the Dutch TV-show "De Kennis van Nu" and in particular Dirk de Bekker, Diederik Jekel, Ines Kaal, Susanne Linssen, Elisabeth van Nimwegen, Roland Vissers, and Marcia van Woensel for the inspiring conversations about our project and the facilitation of the data collection of protocol 2. We want to thank panelists at seminars at the University of Duisburg, researchers (a) only theorize about affect while admitting that they "lack direct measures of somatic response" (Lodge and Taber 2013, 208), (b) study only one dimension of core affect, specifically, arousal (Mutz 2007; Mutz and Reeves 2005; Soroka, Fournier, and Nir 2019; Soroka and McAdams 2015), or (c) study the cognitive corollaries of affect, such as discrete emotions like anger and anxiety (Aarøe 2011; Delton, Petersen, and Robertson 2018; Huddy, Mason, and Aarøe 2015; Klar 2013; Marcus, Neuman, and MacKeun 2000).

We tackle these issues by establishing the circumplex model in political science as a theory of core affect. This theory distinguishes between two dimensions: the intensity of affective responses-arousal-and the direction

University of Southern Denmark, University of Leiden, University of Michigan, University of Nijmegen, The Ohio State University, University of Illinois at Urbana-Champaign, University of Montreal, George Washington University, University of Zurich as well as participants at the International Society for Political Psychology 2017, International Communication Association Meeting 2017, Dutch Political Psychology Meeting 2017, Dutch Communication Association Meeting 2017, Southern Political Science Association Meeting 2018, the Challenges Group at the University of Amsterdam, Amsterdam School of Communication Research and the Hot Politics Lab for the feedback and suggestions. We want to thank Vin Arceneaux, Lene Aarøe, John Bullock, Brian Burgoon, Ted Brader, Claire Gothreau, Kim Gross, Liesbeth Hooghe, John Hibbing, Aleks Ksiazkiewicz, Gary Marks, Yphtach Lelkes, Michael Bang Petersen, Martin Rosema, Kevin Smith, Stuart Soroka, and Catherine de Vries for comments and suggestions during this project. Throughout this project, Ming Boyer, Emke de Vries, Sander Kunst, Judith Meijer, Isabella Rebasso, Denise van de Wetering, and Myrthe Willems provided excellent assistance during the data collection, and we want to thank Bert Molenkamp, Marco Teunisse, and Jasper Wijnen for their technical assistance. Author contributions: designed the study: B.N.B, G.S., \& M.R.; conducted the study: B.N.B., G.S., \& M.R.; data preprocessing: G.S.; data analysis: B.N.B. \& G.S.; writing of the paper: B.N.B., G.S., \& M.R.; revisions of the paper: B.N.B., G.S., \& M.R. Replication materials can be found on Dataverse at: https://doi. org/10.7910/DVN/LCWR7G.

Received: October 14, 2019; revised: March 23, 2020; accepted: May 30, 2020. 
of affective responses-valence (Russell 1980). This enables us to theorize about the structure of affective responses to political rhetoric and to examine this empirically by using physiological measures. We capture changes in arousal using skin conductance and are the first in the discipline to capture valence in response to political rhetoric by means of facial electromyography (EMG). In addition, we assess attention with heart rate variability.

Why study affective responses? According to the "hot cognition hypothesis," all sociopolitical concepts are affectively charged in the brain (Abelson 1963). Political issues-but also leaders and parties-are affectively tagged in our long-term memory. This means that when we retrieve information about an issue from our memory, positive or negative affect is activated automatically. This colors subsequent cognitive evaluations of the situation (Lodge and Taber 2013), and it may motivate expressions of specific discrete emotions such as anger, anxiety, or enthusiasm (Brader 2006).

Existing theories predict variation in affective responses to political rhetoric but assess such affective responses only indirectly. We assess three possible sources of variation in affective responses and the consequences of these affective responses to political rhetoric. First, people with more political knowledge have stronger affective links to political objects (Lodge and Taber 2005) and might, therefore, experience more arousal when exposed to political rhetoric. Second, according to the directional model of voting, the more extreme an attitude is, the more intense the arousal attached to the attitude will be (Rabinowitz and Macdonald 1989). Third, from the motivated reasoning framework we derive that people experience negative affect in response to incongruent information and positive affect in response to congruent information (Lodge and Taber 2013; Suhay and Erisen 2018; Taber and Lodge 2006).

To investigate the variation in affective responses to political rhetoric, we report an unusually large lab experiment $(N=397)$ with physiological measures. To maximize variation on our independent variables of interest-political knowledge, attitude extremity, and attitude position-our experiment was conducted at a university lab and at several other public locations. Participants were randomly exposed to short video clips on with left-wing or right-wing rhetoric on different political issues.

We find that people with extreme prior attitudes on an issue are more aroused than people with less extreme attitudes. We find no statistically significant association between political knowledge and arousal. Also, we report that incongruent rhetoric produces negative affect, whereas congruent rhetoric does not produce positive affect. Finally, we present evidence that arousal predicts issue position change, independent of discrete emotions.

In the conclusion, we set out to integrate our findings regarding the affective component of emotions within the framework that studies the cognitive component of emotions-that is, discrete emotions. We outline a research agenda to theorize the interplay between these different components of emotions and their consequences for politics.

\section{WHAT ARE AFFECTIVE RESPONSES TO POLITICS?}

Affect is a part of the larger concept of emotion. One can define emotions as "episodic, relatively short-term, biologically based patterns of perception, experience, physiology, action, and communication that occur in response to specific physical and social challenges and opportunities" (Keltner and Gross 1999, 468). Affective responses are the immediate physiological responses to stimuli. These responses are uncontrollable and occur between 50 and 100 milliseconds after receiving a stimulus. This is prior to cognitive evaluations, which commence after 500 milliseconds (Lodge and Taber 2013).

We define affective responses following the circumplex model of affect (Russell 1980). This distinguishes between two affective dimensions: arousal and valence. Arousal captures the intensity of affect and is measured with skin conductance. Valence captures the direction (positive or negative) of affect and is measured with facial EMG. These measures are rare in political science. Some studies analyze differences between liberals and conservatives in arousal to negative stimuli (Arceneaux, Dunaway, and Soroka 2018; Oxley et al. 2008). In these studies, arousal is an independent variable and considered a trait (for a critique of this approach, see Bakker et al. 2020). Our paper is more in line with work that studies arousal as a response to political communication (Mutz 2007; Mutz and Reeves 2005; Soroka, Fournier, and Nir 2019; Soroka and McAdams, 2015).

We move beyond arousal by turning to valence as well. This dimension of affect is largely absent in political science. This is remarkable because there are various canonical theories within the field that hint that political rhetoric can evoke both positive and negative affect (Soroka 2014; Taber and Lodge 2006). The core contribution of our study is that by introducing the circumplex model of affect we can theorize about arousal and valence and at the same time offer ways to measure this by using facial EMG.

How do affective responses align with the more cognitive discrete emotions? This is a big unsettled question in neuroscience (Barrett and Satpute 2019; LeDoux and Pine 2016). Subjective appraisals of affect are typically, but not always, translated into concrete discrete emotions such as anxiety, anger, or enthusiasm. Affect-sometimes labeled "core affect" - is thus causally prior to the appraisal of (discrete) emotions (Barrett and Satpute 2019), and it requires a different methodology to measure. This means that our work is different from seminal work in political science research on emotions, which uses self-reported discrete emotions (e.g., Aarøe 2011; Delton, Petersen, and Robertson 2018; Huddy, Mason, and Aarøe 2015; Klar 2013; Marcus, Neuman, and MacKeun 2000).

Self-reported emotions can align with affect (Bradley et al. 2001). Within the circumplex model, anger is, for instance, seen as a discrete emotion that is characterized by high arousal and negative valence (Russell, 1980). Yet the correlation between (physiological) affect and self-reported emotions is typically very low (LeDoux and 
Pine 2016). This might be because they are produced by different brain systems. That said, physiology and cognitive-emotional responses are both part of the broader concept of emotion. It is not wrong to study one or the other; they are simply different, and they may have different consequences. We investigate these consequences in this paper by also analyzing whether selfreported discrete emotions and physiological responses can explain attitude change. In addition to that, in our conclusion we set out a research agenda that can further explore the relevance of studying emotions as both unconscious, physiological responses and conscious, self-reported emotions. Before we continue to specify our expectations about physiological affective responses, below we discuss each measure in more detail.

\section{Arousal}

Skin conductance captures activity of the sympathetic nervous system (Dawson, Schell, and Filion 2007). It measures the varying electrical properties of the skin in response to the increase of sweat secretion in the eccrine glands. With more sweat secretion, the conductance of electricity improves and skin conductance levels rise. This is interpreted as an increase in arousal. Increases in skin conductance have been reported in response to negative images (Lang et al. 1993), negative news (Soroka, Fournier, and Nir 2019; Soroka and McAdams 2015), and negative political ads (Daignault, Soroka, and Giasson 2013; Wang, Morey, and Srivastava 2014), but such increases are also observed in response to positive stimuli such as a exposure to a preferred football team (Potter and Keene 2012), political party (Petersen, Giessing, and Nielsen 2015), or politician (Wagner et al. 2014).

\section{Valence}

The valence of affect can be measured by recording electromyographic (EMG) signals of specific muscles in the face (Tassinary, Cacioppo, and Vanman 2007). To disentangle the experience of negative and positive affect, the activity of the corrugator and zygomaticus muscle groups, respectively, are recorded (Tassinary, Cacioppo, and Vanman 2007). The corrugator ciculii is a muscle above the eyebrow that draws the brow down and pulls the brows together. Corrugator activity has been recorded in response to negative images (Lang et al. 1993), negative words (Wexler et al. 1992), and negative affective cues in language (Hietanen, Surakka, and Linnankoski 1998). The zygomaticus major pulls the corners of the mouth up and back into a smile (Larsen, Norris, and Cacioppo 2003). Zygomaticus activity increases in response to positively valenced images (Van Oyen Witvliet, and Vrana 1995) and videos (Cacioppo et al. 1986).

\section{Attention}

A fourth physiological measure that is sometimes used is heart rate variability. In some cases it is used to measure arousal, and in other cases it is used to measure (cognitive) attention (Lang, Newhagen, and Reeves 1996; Soroka, Fournier, and Nir 2019; Soroka and McAdams 2015). However, any acceleration in heart rate that comes from arousal will be overwhelmed by the deceleration that comes with attention (Potter and Bolls 2012). We follow Soroka and colleagues (Soroka, Fournier, and Nir 2019; Soroka and McAdams 2015) and employ heart rate variability as a measure of attention.

\section{WHO HAVE STRONGER AFFECTIVE RESPONSES TO POLITICS?}

It seems unlikely that all political issues are equally "hot" for all citizens. Brader $(2006,187)$ pointed out that the next generation of studies "must consider" how "individual variation" conditions the extent to which people experience affect towards politics. As such, we move this literature one step further and distinguish three sources of variation: knowledge, attitude extremity, and (in)congruence with political rhetoric.

According to the "hot cognition hypothesis," all sociopolitical concepts are affectively charged in the brain (Abelson 1963). In our long-term memory we have stored associations between an object and a good, bad, or ambivalent evaluation. Leaders, parties, and even issues can be such objects, and when exposed to them, we quickly and unconsciously retrieve the relevant affective tags from memory (Lodge and Taber 2013). Experimental priming tasks indeed demonstrate the affective nature of the retrieval of political objects from long-term memory (Erisen, Lodge, and Taber 2014; Lodge and Taber 2005). Yet, people differ in the chronic accessibility of evaluations (Lau 1989) because the strength of the associations between objects and their evaluation differs. Repeated evaluations are likely to strengthen these associations; therefore, they make affective tags more chronically accessible. People with more political knowledge are more likely to have "thought about and repeatedly evaluated" political concepts than are people with little to no political knowledge (Lodge and Taber 2005, 471). The more knowledgeable "have formed crystallized attitudes to a fuller set of political issues" because of their "interest in politics" (Lodge and Taber 2005, 473). Therefore, it is plausible that people with more political knowledge have formed stronger affective links to political objects (Lodge and Taber 2005). Indeed, more politically sophisticated citizens self-report stronger discrete emotions towards politicians in surveys (Brader 2006). Hence, for people with high levels of political knowledge, political rhetoric is more likely to be arousing because the concepts activated by this rhetoric are more accessible than is the case with people with little political knowledge. This leads us to expect that the more political knowledge someone has, the more s/he experiences arousal in response to political rhetoric $(\mathbf{H 1})$.

A second factor that might evoke affective responses is attitude extremity. The theory of directional voting (Rabinowitz and Macdonald 1989) attributes a central role to affect because political issues are affectively charged. Thus, "for issues (or other 
political cues) to have impact, they must evoke emotions" (Rabinowitz and Macdonald 1989, 94). Because people differ in their attitudes to these issues, the intensity of affective responses will differ per individual. Neutral (indifferent) attitudes are cold (no arousal), while the more extreme an attitude becomes, the more intense the affect (arousal) attached to the attitude. Such thinking is in line with the motivated reasoning framework. Stronger (more extreme) attitudes on an issue produce stronger biases in information processing (Lodge and Taber 2013). Affective primes also have stronger effects on people with stronger attitudes on an issue (Lodge and Taber 2005). The underlying rationale is similar to that of our previous hypothesis concerning political knowledge. People with strong attitudes are more invested in an issue, and they have given it more thought. Therefore, these concepts are more chronically accessible. Also, the affective tags that are activated are more likely to be exclusively positive or negative for people with strong opinions on an issue. In sum, we expect that the more extreme someone's attitude on an issue is, the more s/he experiences arousal in response to political rhetoric on that issue $(\mathbf{H 2})$.

A third possibility is that not issue extremity but the extent to which someone's issue position is congruent with the political message s/he is exposed to conditions affective responses. Here we move from a focus on "arousal" to "valence" because Taber and Lodge (2006) theorized that citizens respond with negative affect to information they disagree with. As a reaction, counterarguments are formulated to reject the attitudinally incongruent message and to reduce the negative affect (Taber and Lodge 2006). There is some indirect evidence that people indeed experience affect in response to incongruent messages. Using a priming paradigm, Lodge and Taber (2005) show that participants produced faster evaluations when the prime was congruent with the target word than when the prime was incongruent with the target word. For a participant identifying as a Democrat, a congruent prime is, for example, Clinton combined with a positive word. An incongruent prime would be Clinton combined with a negative word. These studies led Lodge and Taber $(2005,467)$ to conclude that affect is "triggered automatically on the mere presentation of the concept." Along these lines, preliminary work in neuroscience suggests that when a person disagrees with a message, a strong brain response is evoked (Morris et al. 2003) and brain regions associated with the experience of negative affect appear to be more active (Kaplan, Gimbel, and Harris 2016). We therefore expect that the more incongruent someone's attitude is with the rhetoric s/he is exposed to, the more s/he experiences negative affect (H3). Rhetoric can, of course, also be congruent with prior attitudes. If this is the case, we expect that the more congruent someone's attitude is with the rhetoric s/he is exposed to, the more s/he experiences positive affect $(\mathbf{H 4})$. Note that we do not formulate directional expectations about the relationship between incongruence and arousal or congruence and arousal because exposure to incongruent messages and congruent messages could be equally arousing.

Following the argument that incongruent rhetoric motivates people to look for counter arguments (Lodge and Taber 2013), we also expect that the more incongruent someone's attitude is with the rhetoric s/he is exposed to, the more attentive s/he will be (H5). As discussed in the previous section, heart rate variability is a physiological measure of attention, which we use in our study.

Finally, we ask the question of whether affect is associated with subsequent opinion change. Taber and Lodge (2006) demonstrated that people with extreme attitudes and high knowledge were not only reasoning in biased ways, their attitudes also polarized at the end of the study. We have theorized that such biased reasoning is triggered by affect. This raises the question of whether affective responses then also lead to attitude changes. There is, to our best knowledge, no earlier work that suggests how exactly affect and attitude change would be connected. Increased affect could lead to changes in attitudes in different directions: one could move either towards or away from the message one receives. Hence, although we expect that increased affect in response to political rhetoric is associated with attitudinal changes, we do not have clear expectations regarding the specific direction of such changes.

\section{RESEARCH DESIGN}

\section{Sample}

Our study was conducted at multiple locations in the Netherlands between August 2016 and June 2017. We used two different protocols. Table 1 describes the locations, specifies the protocols used, and notes the number of participants collected at each location. In all we have collected data for 397 Dutch adults. No statistical methods were used to predetermine sample size, but our sample size is more than three times larger than the median sample size reported in previous publications (e.g., Arceneaux, Dunaway, and Soroka 2018; Mutz 2007; Mutz and Reeves 2005; Renshon, Lee, and Tingley 2015; Soroka, Fournier, and Nir 2019; Soroka and McAdams 2015)-see Appendix A.1 for more details. Our study thus has an unusually large sample size for a study of its kind.

To obtain sufficient variation on the theoretically relevant independent variables (e.g., political knowledge and political attitudes), we set up our lab at different lab-in-the-field locations. In Appendix A.4, we demonstrate that we have succeeded in obtaining sufficient variance on our key independent variables.

\section{Design of the Study}

Upon signing the informed consent form, participants completed a survey on a desktop computer (laboratory) or iPad 2 (lab-in-the-field). We asked about their political attitudes and socioeconomic background (see Appendix A.2 for details on rewards and procedures 
TABLE 1. Description of the Locations

\begin{tabular}{|c|c|c|c|}
\hline Location & Description & Issues & $\mathrm{N}$ \\
\hline $\begin{array}{l}\text { Cultural festival } \\
\text { (August 2016) }\end{array}$ & $\begin{array}{l}\text { Lowlands is a three-day cultural festival with a } \\
\text { science program (Lowlands Science) where } \\
\text { scholars can conduct experiments. }\end{array}$ & $\begin{array}{l}\text { Immigration, redistribution, } \\
\text { and climate (protocol.1) }\end{array}$ & 143 \\
\hline $\begin{array}{l}\text { Laboratory (November- } \\
\text { December 2016) }\end{array}$ & $\begin{array}{l}\text { Social science laboratory of a Dutch University } \\
\text { with a participant pool of students and people } \\
\text { living in the vicinity. }\end{array}$ & $\begin{array}{l}\text { Immigration, redistribution, } \\
\text { and climate (protocol 1) }\end{array}$ & 120 \\
\hline Media museum (April 2017) & $\begin{array}{l}\text { Dutch media museum (Beeld \& Geluid). We set } \\
\text { up our lab in the exhibition area. }\end{array}$ & $\begin{array}{l}\text { Immigration, climate, } \\
\text { and EU (protocol 2) }\end{array}$ & 47 \\
\hline $\begin{array}{l}\text { Evangelical Rock Festival } \\
\text { (June 2017) }\end{array}$ & $\begin{array}{l}\text { The EO Jongerendag is a one-day festival for } \\
\text { young adults. We set up the lab at the } \\
\text { information market. }\end{array}$ & $\begin{array}{l}\text { Immigration, climate, } \\
\text { and EU (protocol 2) }\end{array}$ & 33 \\
\hline Biker event (June 2017) & $\begin{array}{l}\text { TT-Assen is a large biker event with multiple } \\
\text { motorbike races. The laboratory was set-up } \\
\text { for one day close to the entrance. }\end{array}$ & $\begin{array}{l}\text { Immigration, climate, } \\
\text { and EU (protocol 2) }\end{array}$ & 14 \\
\hline Fair (July 2017) & $\begin{array}{l}\text { Largest fair (Tilburgse Kermis) in the Netherlands. } \\
\text { We set up the laboratory for two days on the } \\
\text { terrain of the fair. }\end{array}$ & $\begin{array}{l}\text { Immigration, climate, and EU } \\
\text { (protocol 2) }\end{array}$ & 34 \\
\hline
\end{tabular}

during the survey as well as item wording of the survey). Next, participants were connected to physiological measurement equipment by trained research assistants. Participants were also given noise-canceling headphones (Bose). The experiment started with a baseline measure of 30 seconds (Soroka and McAdams 2015). Afterwards, participants were randomly assigned to a treatment in the form of a video clip (between 50 and 60 seconds) about a specific political issue - namely, immigration (protocol 1 and 2$)^{1}$, climate change (protocol 1), redistribution (protocol 1), and the European Union (protocol 2) - see Table 1 for an overview. ${ }^{2}$ We chose these issues because they differ in their salience, with immigration the most salient issue at the time of the study and climate, redistribution, and Europe considerably less salient (den Ridder et al. 2016).

The video clips were created by a professional editor, using a selection of clips from news broadcasts that illustrated these issues, without showing politicians, political parties, or other prominent persons. Per issue, we constructed two treatments: a "left-wing" message (liberal) and a "right-wing" message (conservative). To do this, a professional speech actor recorded a voiceover loosely based on real speeches from left-wing or right-wing parliamentarians. By using real speeches, we can guarantee a degree of external validity, without mentioning the source to the participant, which could bias our treatment effects. Table 2 provides snippets of all messages (see Appendix A.6 for transcripts, links to the videos, and other details of the treatments). In a

\footnotetext{
${ }^{1}$ The video footage and wording of the immigration message differed slightly between protocol 1 and 2 . See Table A.3 (protocol 1) and Table A.4 (protocol 2) in Appendix A.6 for differences in item wording.

${ }^{2}$ We also exposed people to a message about the political establishment. We refrain from reporting the results for that part of the study in this paper because that part of the study is part of a larger project on populism. See Appendix A.5 for a complete description of measures that we collected but did not use in this study.
}

pretest $(N=23)$, we confirmed the ideological slant of the messages (Appendix A.6).

We employ block-randomization whereby the order of the four issues was randomized and for each issue participants were randomly exposed to left-wing or right-wing rhetoric. This means that participants saw a total of four clips. The interstimulus intervals between the different messages were roughly 30-60 seconds, during which participants answered a set of filler questions. Note that we refrain from treating these periods as interstimulus intervals in the analyses because participants also answered questions during this period. As a consequence, their hands moved a lot, causing all sorts of unknown distortions in the measure of the physiological responses.

\section{Survey Measures}

In the survey before the treatment, we measured attitudes towards four issues: (1) immigration (three items, e.g., "The Dutch culture is threatened by refugees" [reverse coded]), (2) redistribution (two items, e.g., "Income inequality is too big in the Netherlands. People with the lowest incomes should get the biggest salary increase"), (3) the EU (three items, e.g., "I am in favor of more decision-making at the European level"), and (4) climate (three items, e.g., "There are more important things in life than protecting the environment" [reverse coded]). All variables were measured on a 9-point scale ranging from "totally disagree" (1) to "totally agree" (9)-see Appendix A.7 for item wording. Descriptive statistics for these and all other measures in this study are provided in Appendix A.3. After each treatment, respondents were asked again to fill out these questions. For our final analysis of the consequences of affective responses, we use the absolute difference between the pretreatment and posttreatment measure as the dependent variable. Attitude extremity on an issue was measured by taking the absolute distance between the participant's placement on the scale from 
TABLE 2. Treatments: Snippets of the Message

\begin{tabular}{|c|c|c|}
\hline Issue & Left-wing & Right-wing \\
\hline Immigration & $\begin{array}{l}\text { "The Netherlands must be a safe haven for } \\
\text { people fleeing from war and violence. Don't } \\
\text { stop refugees, but take them in!" (55 seconds) }\end{array}$ & $\begin{array}{l}\text { "Our security, freedom, our culture, our money } \\
\text { and our future are at stake. ... It is enough. } \\
\text { The borders must be closed!" (55 seconds) }\end{array}$ \\
\hline Redistribution & $\begin{array}{l}\text { "It is about time that the situation of people on } \\
\text { lower incomes improves. The government } \\
\text { must tax the rich more heavily." ( } 47 \text { seconds) }\end{array}$ & $\begin{array}{l}\text { "Reducing income differences by raising extra } \\
\text { taxes ... is theft. Hardworking people should } \\
\text { never be the victim of an overpowering } \\
\text { government." ( } 41 \text { seconds) }\end{array}$ \\
\hline EU & $\begin{array}{l}\text { "Together, Europe is strong. Therefore: more } \\
\text { Europe!" (48 seconds) }\end{array}$ & $\begin{array}{l}\text { "It is about time to liberate the Netherlands from } \\
\text { the claws of the EU-super-state. It is time to } \\
\text { take back control." ( } 50 \text { seconds) }\end{array}$ \\
\hline Climate & $\begin{array}{l}\text { "The Netherlands should now invest and switch } \\
\text { to sustainable forms of energy. . . This is the } \\
\text { only way we can save our planet. We don't } \\
\text { have a second to lose." (65 seconds) }\end{array}$ & $\begin{array}{l}\text { "Investing billions in . . green socialism to solve } \\
\text { a barely defined problem, is an appalling } \\
\text { human arrogance. No more penny for all the } \\
\text { nonsensical climate policy." (53 seconds) }\end{array}$ \\
\hline
\end{tabular}

Note: Complete text of treatments and links to videos can be found in Appendix A.6.

FIGURE 1. Physiological Measures: SCL and EMG

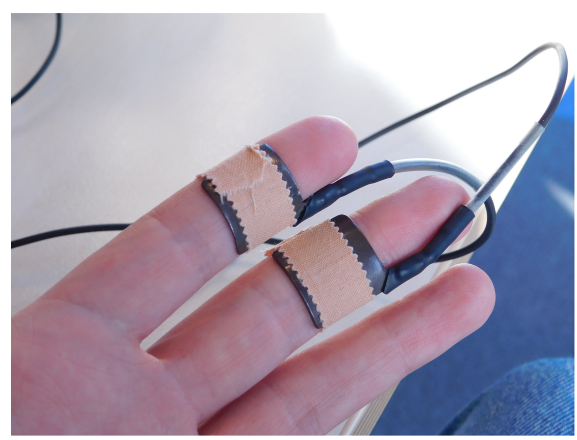

[SCL]

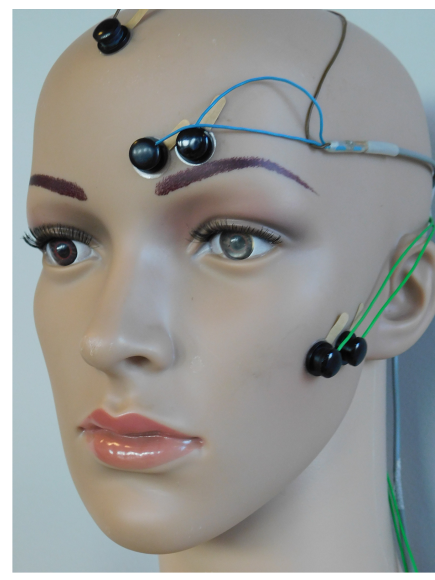

[EMG] the scale's middle point. We measured political knowledge using three multiple choice items, such as "How long is one term for a member of the Lower House" (correct answer $=4$ years; see Appendix A.7 for other items and psychometric properties). We created an index by summing the correct answers.

For all four issues, we created an index of incongruence by contrasting the pretreatment attitudes with the video clip participants received. Consider immigration attitudes. A score from $>0$ to +4 means that the video clip participants received was incongruent with their immigration attitudes (left-wing attitude with rightwing clip or right-wing attitude with left-wing clip). The higher the score, the more incongruent the clip was with the attitude. Scores $<0$ to -4 indicate congruence between the video clip and the attitude (left-wing attitude and left-wing clip, etc.). Again, we assume that the message is more congruent for participants with more extreme attitudes. A score of " 0 " indicates that participants have a neutral immigration attitude, and so neither the left-wing nor the right-wing video clip is incongruent with their attitude.

\section{Physiological Measures}

We recorded physiological data using the Versatile Stimulus Response Registration Program 1998 (Vsrrp98) software on laptops (lab-in-the-field data collection) or stationary computers running Windows 7 (laboratory data collection). We assess arousal using skin conductance levels (SCL). These are measured by passing a small current through two electrodes placed on the skin (see panel A of Figure 1). By keeping the current constant, it is possible to measure the flow of the current - what we call skin conductance expressed in micro-Siemens (Dawson, Schell, and Filion 2007). For a description of the measure, see Appendix A.8. 
We constructed our measure of change in skin conductance response $\left(\triangle \mathrm{SC} L_{i}\right)$ by taking the difference between the logged mean SCL recorded while participants were exposed to treatment $T$ and the logged mean SCL recorded during the baseline. To minimize the effects of extreme values, we follow Oxley et al. (2008) and take the average of the natural log of SCL during the image and the ISI (Equation 1 summarizes this approach). As such, we create a change-inskin-conductance-level variable whereby higher values indicate that skin conductance levels increase during the exposure to the message.

$$
\Delta S C L_{i}=\frac{\sum_{j=1}^{\text {time }_{T}} \ln \left[S C L(T)_{\mathrm{ij}}\right]}{\text { time }_{T}}-\frac{\sum_{j=1}^{\text {time }_{T}} \ln \left[S C L\left(\text { Baseline }_{i j}\right]\right.}{\text { time }_{T}}
$$

The valence of emotions is measured using a facial electromyagram (EMG) (Tassinary, Cacioppo, and Vanman 2007; see Appendix A.10 for more details). ${ }^{3}$ To measure negative affect, we measure the activity of the corrugator supercilii muscle-above the eyebrow (see panel B of Figure 1). The corrugator has no overlapping muscle groups and has a very limited representation in the motor cortex and "tends to be bilateral innervated" (Larsen, Norris, and Cacioppo 2003, 776-777). The measurement of the corrugator is therefore less subject to disruptions from the (voluntary) movement of other muscles.

To measure positive affect, we measure the activity of the zygomaticus major (Larsen, Norris, and Cacioppo 2003). It is a difficult muscle to measure because it has greater contralateral innervation (Larsen, Norris, and Cacioppo 2003), and the cheek - where the zygomaticus is located (see panel B of Figure 1) -is a particularly crowded area of the face with lots of muscles (Tassinary, Cacioppo, and Vanman 2007). This makes measures of the zygomaticus susceptible to "cross talk" (Larsen, Norris, and Cacioppo 2003, 777). Irrespective of the difficulties of measuring the zygomaticus, it is a unique measure to capture positive affect. Zygomaticus activity was only recorded in protocol 1 and not in protocol 2.

People do not constantly frown or laugh, at least not in response to the type of political messages we used during the experiment. The object of interest of EMG analyses is whether people frowned or laughed. To evaluate this, we first cleaned the data as follows: we extracted EMG per second and calculated the change between $t$ and $t-$ 1. There was a time trend in that data. We extracted the time trend by regressing change on time. The subsequent model predictions were subtracted from the transformed data. We subsequently decided on a cutoff value of

\footnotetext{
${ }^{3}$ The lab equipment we used was able to reliably and validly capture facial EMG activity in earlier work in other domains, see for instance Gazendam, Kamphuis, and Kindt (2013), Nohlen et al. (2016), Rotteveel et al. (2001), and Sevenster, Beckers, and Kindt (2012).
}

10 microvolts as a peak. We kept all increases of 10 microvolts and larger, and for each individual we summed these peaks and logged it to procure a normally distributed variable. We have experimented with several alternatives: using individual cutoff values based on withinindividual deviations, sums of all changes, and the number of peaks. In most cases, correlations between the 10-microvolt cutoff point and the other variables were high, and the alternative operationalizations led to similar regression output (see Appendix C.2). ${ }^{4}$

Like all other physiological measures, heart rate variability was registered with the VSRRP98 program (see Appendix A.9). The program contains an algorithm to extract peaks from the raw heartbeat data. We manually checked and corrected the algorithm where necessary. The program also calculates the root mean square of successive differences (RMSSD), which is a standard measure of heart rate variability - that is also used by Soroka, Fournier, and Nir (2019) - and is based on the standard deviation of the interbeat intervals (Blascovich et al. 2014). As we do for skin conductance, we compare the mean heart rate variability during exposure to the message with the baseline. A positive value indicates an increase in heart rate variability compared with the baseline, while a negative value indicates a decrease in heart rate variability.

All physiological measures were visually inspected by the researchers as well as a student assistant blind to the expectations of our study. We cross-checked anomalies with the lab log. In the case of EMG, sometimes electrodes fell off during the experiment, and these data points were removed. In the case of heart rate there were some anomalies due to movement of the participant or improper placement of the measurement equipment. We also removed these data points. As a consequence, the number of observations will differ per analysis of physiological measures.

In a subset of protocol $1-$ during the cultural festival (see Table 1$)$-we asked respondents $(N=143)$ to self-report their levels of enthusiasm, anger, and anxiety on a $0-100$ thermometer in response to the immigration treatment. ${ }^{5}$ In this subset of our data, these measures do not correlate with the physiological measures. ${ }^{6}$ We use these self-reported emotions in the final exploratory analysis concerning the effects of physiological responses (see Appendix A.11 for all correlations between physiology and self-reported emotions).

\footnotetext{
${ }^{4}$ Other alternatives were not used because they had some conceptual problems. Individual deviations are problematic if individuals do not laugh or frown at all. Deviations are then only based on the static produced in the EMG measuring process. Similarly, the sum of all changes takes into account irrelevant statics.

${ }^{5}$ We could not ask this more often as the lab-in-the-field design required us to keep the protocol short to limit the costs (time) for participants.

${ }^{6}$ Anger-Arousal (SCL): $r=0.02$, Anxiety-Arousal: $r=-0.07$, Enthusiasm-Arousal: $r=-0.03$; Anger-Negative valence (Corrugator) $r=0.08$, Anxiety-Negative valence $r=-0.17$, Enthusiasm-Positive valence $($ Zygomaticus $)=0.001$.
} 


\section{Method of Analysis}

To evaluate our hypotheses, we pool the data across the different clips $(N=961$ for arousal, $N=1,032$ for negative affect, $N=1,012$ for heart rate variability, and $N=429$ for positive affect). We pool because of power reasons. Similar to earlier psychophysiological studies (e.g., Mutz 2007; Mutz and Reeves 2005; Soroka, Fournier, and Nir 2019; Soroka and McAdams 2015), we expected small-sized effects. A power analysis conducted after the data was collected and based on the regression procedure described below indicates that our achieved power in the pooled analysis of corrugator activity is 0.96 . However, once we subset to specific issues, specific clips, or on independent variables, power drops dramatically to values below 0.8 (see Appendix A.1 for calculations). Therefore, we are reluctant to disaggregate our analyses to the individual clips or other subsets of the data.

For Hypotheses 1 and 2, we run one OLS regression analysis: our dependent variable is change in skin conductance and our main independent variables are attitude extremity and political knowledge. In this analysis, we control for the computer the respondent used, the temperature during the experiment (Venables and Mitchell 1996), the order in which the treatment was received, the salience of the issue (for the respondent), gender, age, educational level, the percentage level of alcohol in blood, and dummy variables to pick up differences between treatments. We provide detailed information about each of the covariates in Appendix A.7. By using these covariates, we pick up any differences in the protocols as well as possible distortions due to the time and place of the specific data collection. We cluster our standard errors by individual to account for the interdependence in our observations. Formula 2 is used to evaluate hypotheses 1 and 2, with $k$ reflecting the issue and Right-wing reflecting whether participant ${ }_{i}$ was assigned to the right-wing treatment on issue $k$ (1) or the left-wing treatment on issue $k(0){ }^{7}$

$$
\begin{aligned}
& \Delta \mathrm{SC} L_{i k}=\beta_{0}+\beta_{1} * \text { Extremity }_{i k}+\beta_{2} * \text { Knowledge }_{i} \\
& +\beta_{3} * \text { Right-wing }{ }_{i k}+\text { controls }
\end{aligned}
$$

For the hypotheses $3-5$, we add the incongruence variable and omit the treatment direction variable. This leads to formula 3 . We use the change in corrugator activity, the zygomaticus activity, and heart-rate variability (RMSSD) as dependent variables.

$$
\begin{gathered}
\Delta \text { Corrugator }_{i k} / \Delta \text { Zygomaticus }_{i k} / \Delta \text { RMSSD }_{i k} \\
=\beta_{0}+\beta_{1} * \text { Extremity }_{i k}+\beta_{2} * \text { Knowledge }_{i}+\beta_{3} \\
* \text { Incongruence }_{i k}+\text { controls }
\end{gathered}
$$

For the purposes of interpretation we have standardized all variables, except for the dichotomous variables.

\footnotetext{
${ }^{7}$ Covariates are not highly correlated with each other so we do not need to worry about potential multicollinearity issues in these models. See Appendix A.11 for a correlation matrix.
}

\section{RESULTS}

To get a feel for the data, we have first plotted the means and $95 \%$ confidence intervals of the four physiological measures per clip (see Figure 2). There is considerable variation across issues regarding the level of arousal (see upper left panel). In particular, the Immigration (protocol 1) and the left-wing Climate clips stand out as having lower than baseline levels of arousal. This means that fewer people were aroused when exposed to these clips. Specifically, about $70 \%$ of the participants were aroused by the Immigration protocol 2 clips, and only $50 \%$ of the participants were aroused by the Immigration protocol 1 clips. The two valence measures (bottom row Figure 2) have relatively similar values across issues and left-wing and right-wing clips. There is, on average, a bit more corrugator and zygomaticus activity with right-wing clips than with left-wing clips. For RMSSD - the attention measure (see upper right panel) - the mean values are negative. This indicates that heart-rate variability slows down when people concentrate. In most cases the mean is rather similar, but for the left-wing EU and Immigration (protocol 2) clips, the heart-rate variability is somewhat higher (therefore less attention).

\section{Attitude Extremity, Knowledge, and Arousal}

Now we evaluate hypotheses 1 and 2. Arousal is the dependent variable, and it is measured as the difference in skin conductance between the treatment and the baseline. Figure 3 plots the effects of attitude extremity, political knowledge, and the control variables. The effect of political knowledge (H1) is positive but not statistically significant $(b=0.034, \mathrm{SE}=0.047, p=0.466)$. The effect of attitude extremity (H2) is positive and statistically significant $(b=0.073, \mathrm{SE}=0.034, p=$ 0.031). ${ }^{8}$ Therefore, we reject $\mathrm{H} 1$ while accepting $\mathrm{H} 2$. The effect of extremity is small - but with the exception of the gender and education covariates - it is the only significant individual-level variable. The plots on the right side of Figure 3 demonstrate that the effect of extremity is similar when analyzing the full exposure to the treatment or just the first 10 to 20 seconds of exposure. We do find significant differences in arousal between issues. Compared with the climate issue, the EU issue produces more arousal. Interestingly, immigration is more arousing than climate in the second protocol and less arousing than climate in the first protocol. If we split our analyses per issue (see Appendix C.1), the effects of extremity remain positive, but they are not always significant due to low power. The effects of political knowledge do differ in direction depending on the issue. For example, political knowledge has a positive effect for the inequality issue but a

\footnotetext{
${ }^{8}$ Excluding attitude extremity from the model used to test H1 does not change the effect of political knowledge $(b=0.030, \mathrm{SE}=0.032$, $p=0.349$ ). This is not surprising, as political knowledge and attitude extremity are basically uncorrelated with each other $(r=-0.018,95 \%$ CI $[-0.078,0.043])$. For the full matrix of correlation coefficients between all variables included in our models, see Table A.9.
} 


\section{FIGURE 2. Mean Levels of Arousal and Valence Per Clip}
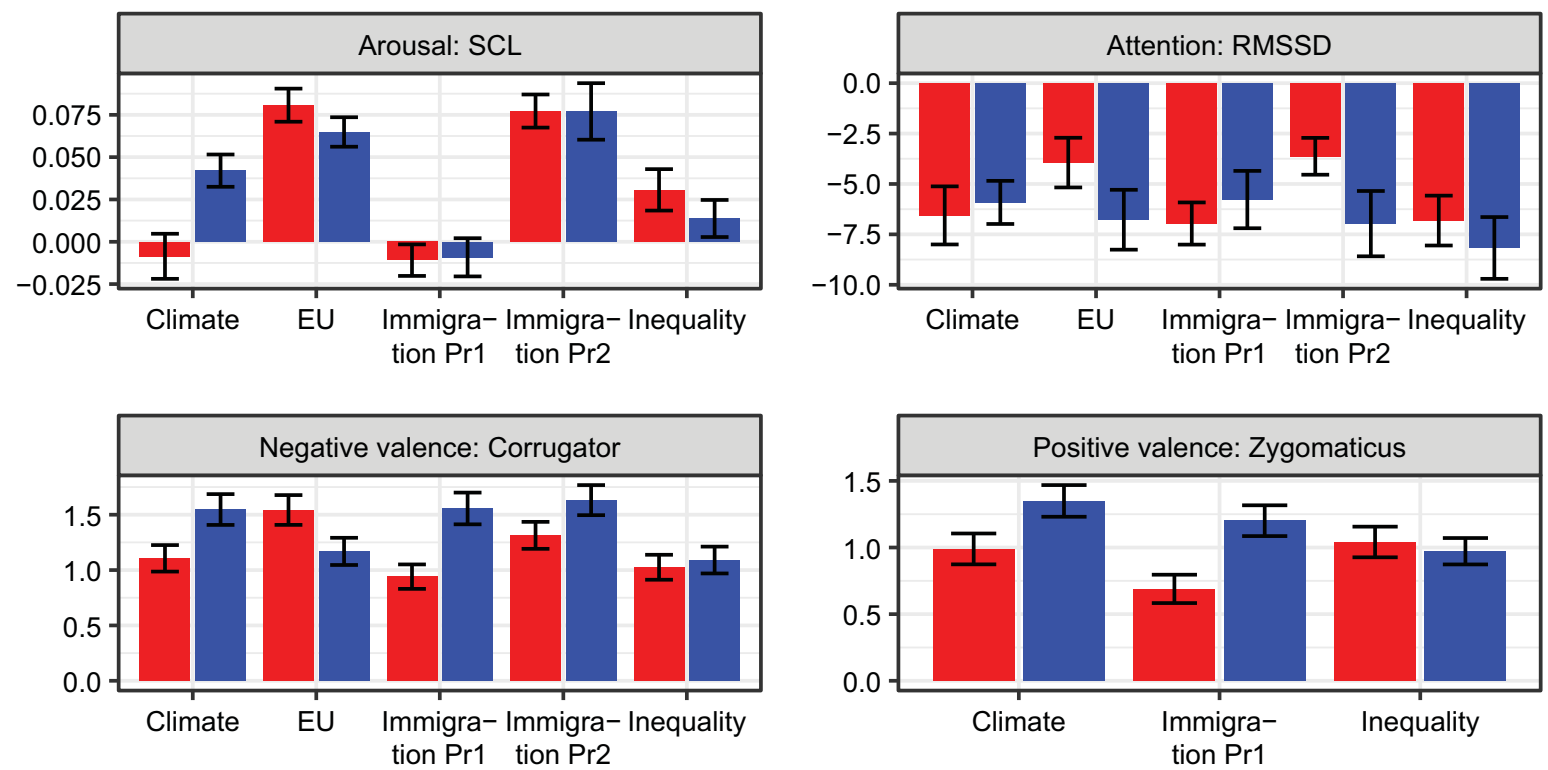

Left-wing clip

Right-wing clip

Note: The thick bars are the means per group; the thin bars are the $95 \%$ confidence intervals.

FIGURE 3. Effects of Attitude Extremity and Political Knowledge on Change in Arousal in Response to Political Rhetoric about Immigration, Redistribution, EU, and Climate
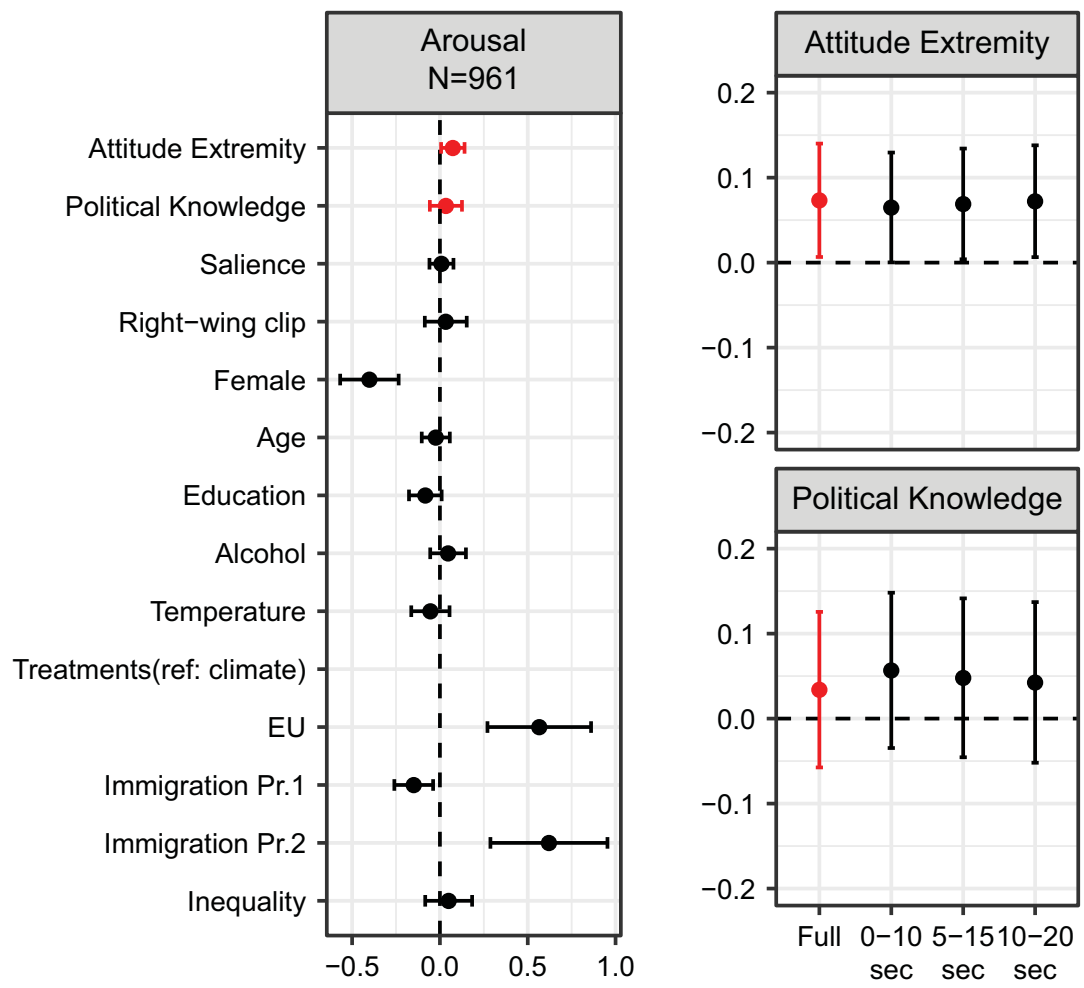

Note: See Appendix B, Table B.1 for the regression output. We have omitted the differences between computers coefficient here. 


\section{FIGURE 4. Physiological Responses to Attitudinal Incongruent Political Rhetoric}

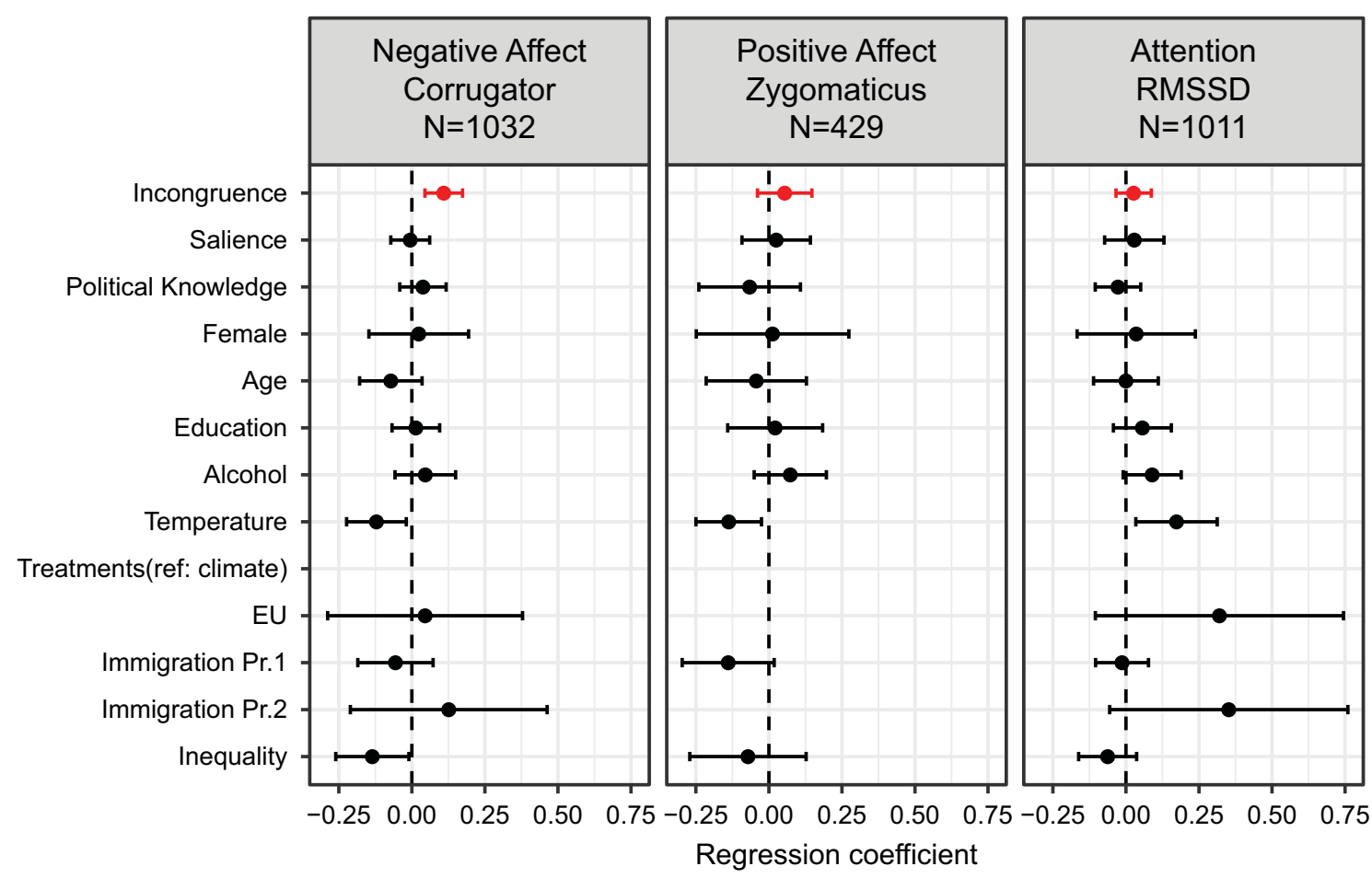

Note: See Appendix B, Table B.2 for the output of the regression analyses. We have omitted the differences between computers coefficient here.

negative effect for the immigration issue (protocol 2). We have no strong explanation of why these differences in arousal per issue occur, but we discuss various possible reasons in the discussion section. The "rightwing" variable is insignificant in all our analyses, so there are no differences in arousal between left-wing and right-wing video clips.

\section{Physiological Responses to Incongruent and Congruent Political Rhetoric}

To test H3-H5, we ran a new series of OLS regression models. Here negative valence (H3, corrugator), positive valence ( $\mathrm{H} 4$, zygomaticus), and attention ( $\mathrm{H} 5$, heart rate variability) are the dependent variables. We again pool the data and analyze each physiological dependent variable (corrugator, zygomaticus, and heart rate variability) independently. Figure 4 reports the effects of the independent variables in these models (see tables in Appendix B for the regression output). Note that all variables, except for the dichotomous ones, are standardized to facilitate interpretation.

Do incongruent messages evoke negative affect (H3)? We find a positive association between the incongruence of the treatment and corrugator activity $(b=0.109, \mathrm{SE}=0.045, p<0.001)$. This means that as incongruence increases, the activity of the corrugator becomes stronger. None of the other individual-level variables matter. Increases in temperature reduce corrugator activity somewhat. Also, corrugator activity in response to the inequality issue is somewhat lower than with the climate treatment. If we split this analysis per issue, we find that the effect of incongruence is driven by corrugator responses to the inequality and immigration (protocol 1) issues (see Appendix C.1).

Do congruent messages evoke positive affect (H4)? For this we look at the middle panel of Figure 4 and expect a negative sign of the incongruence variable. However, the sign is positive and insignificant. We therefore reject $\mathrm{H} 4$. This null-effect is not driven by a particular issue; all effects are clearly non-negative (see Appendix C.1).

Do incongruent messages evoke lower heart rate variability (H5)? Again, we expect a negative sign of the incongruence variable. However, the sign of the incongruence variable is positive and insignificant (Figure 4 right panel). ${ }^{9}$ Furthermore, we find positive effects for alcohol and temperature and no level differences between issues. Splitting the analyses per issue does not change our conclusion (see Appendix C.1). In sum, we reject H5.

\footnotetext{
${ }^{9}$ We have reanalyzed the data using the mean heart rate variability during the stimulus period (see also, Soroka, Fournier, and Nir 2019; Soroka and McAdams 2015). This variable is different from the change in heart-rate variability variable we used, as the correlation between the two variables is negative $(r=-0.139)$. However, the substantive conclusions remain the same and can be derived from the replication files.
} 


\section{FIGURE 5. Estimates of Absolute Position Change}

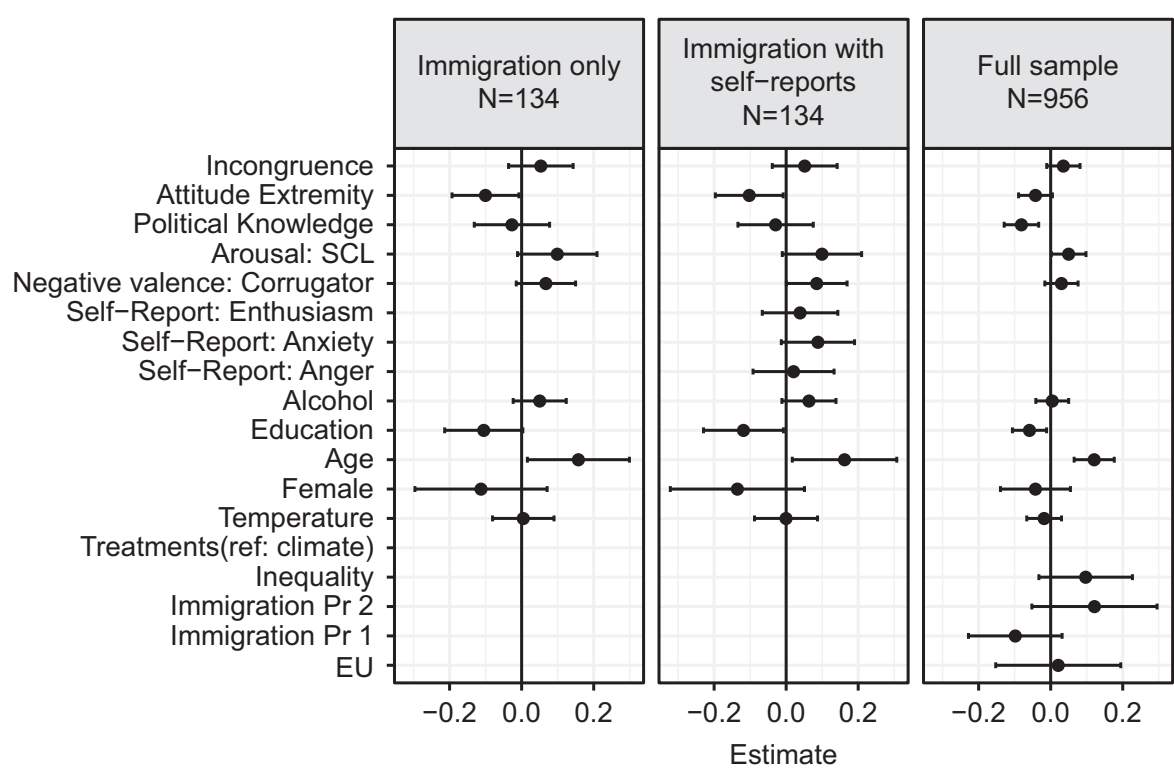

Note: Points are regression coefficients; bars are $95 \%$ confidence intervals.

\section{Consequences of Physiological Responses}

Do these physiological responses have consequences regarding how people position themselves on these issues? We now use the posttreatment issue attitude questions and calculate the absolute difference between pretreatment and posttreatment position per issue. Comparing these positions, approximately $75 \%$ stays very close (less than one point change) to the original position.

Figure 5 presents three OLS regression analyses where absolute position change is the dependent variable. In these models, we have omitted heart rate variability and zygomaticus because we could not explain variation in these variables in our analyses in the previous section. First, we analyze absolute position change for the immigration film (protocol 1 during the cultural festival) only (left panel). We focus on the immigration issue (protocol 1) because we have only asked about self-reported emotions regarding this treatment (see left-hand panel of Figure 5). Attitude extremity reduces absolute position change, which is probably a ceiling effect. Interestingly, both arousal and negative valence have a positive effect on attitude change and are marginally significant $(p<0.10)$. Second, regarding the self-reported emotions, we asked respondents whether they felt enthusiasm, anxiety, or anger during the treatment. Introducing these variables changes the model substantially (see middle panel of Figure 5). The effects of anger and enthusiasm are positive but insignificant, while anxiety has a marginally significant $(p<0.10)$ positive effect. The effect of arousal remains the same, and the effect of negative valence increases in precision $(p<0.05)$. Third, we perform the same analysis for the whole sample (see right-hand panel of Figure 5). Now the self-reported emotions drop out because they were not asked for all issues. The effects of arousal and negative valence become somewhat weaker for the whole sample. Arousal remains positive and significant, whereas the effect of negative valence becomes insignificant. The effect of arousal is similar to Renshon, Lee, and Tingley (2015), who link physiological arousal to stronger anti-immigrant attitudes.

We have also evaluated the presence of mediation effects. We have considered physiological responses as mediators of attitudes (using the full sample) and physiological responses as mediators of self-reported emotions (using the analysis in the middle panel). We have followed the recommendations regarding mediation analysis set out by Imai, Keele, and Tingley (2010). The findings give no indication of mediation effects (see Appendix C.3 for results). Part of this result is also visible when comparing the left and middle panel of Figure 5: the effects of SCL and corrugator barely change after controlling for the self-reported emotions. ${ }^{10}$ As such, the effects of physiological responses seem to be operating independently of self-reported emotions and attitudes.

To conclude the results section, we would like to reflect on the effect sizes we reported. They are small but comparable to existing physiology work. Studies with similar designs and stimulus materials as our study have reported effect sizes between 0.028 and 0.085 (Soroka, Fournier, and Nir 2019; Soroka and McAdams 2015; Wagner et al. 2014) and skin conductance responses to a series

\footnotetext{
${ }^{10}$ We find only a weak indication that the effect of attitude extremity on attitude change is mediated by arousal. As extremity increases attitudes change more once arousal increases-see Appendix C.3.
} 
of validated threatening images between 0.029 and 0.048 (Arceneaux, Dunaway, and Soroka 2018; Bakker et al. 2020). Moreover, small effect sizes are common in political science (e.g., Claassen 2020; Kalla and Broockman 2020; Shepherd and You 2020) and the social sciences in general (Camerer et al. 2018). Finally, small effect sizes are not problematic as long as a study is sufficiently powered to reliably detect small effects-as is the case here.

\section{DISCUSSION}

In this section, we first summarize our findings and core contribution. We continue with a reflection on the unexpected findings. After that we seek to further integrate the affective and cognitive components of emotions in a single theoretical model and set out a research agenda along these lines.

We find that people with more extreme attitudes on an issue experience more arousal when exposed to political rhetoric (H1). Political knowledge, however, does not explain variation in arousal $(\mathrm{H} 2)$. We find that rhetoric that is incongruent with someone's attitudes produces negative affect $(\mathrm{H} 3)$, whereas congruent rhetoric does not produce positive affect (H4). Regarding our last hypothesis, we find no evidence that incongruence increases attentiveness (H5). Finally, we present evidence that arousal and negative affect predict issue position change, independent of self-reported discrete emotions.

The main contribution of this paper is that we establish the two-dimensional circumplex model in political science as a theory of core affect. We connect this model to existing theories in political science and formulate and test how some factors predict arousal in response to political rhetoric while others predict valence. In our study, arousal is stronger as attitudes become more extreme, which aligns with the suggestions from the directional model of voting (Rabinowitz and Macdonald 1989) and motivated reasoning (Lodge and Taber 2013). Negative valence increases as disagreement with rhetoric increases, which provides evidence for the importance of the valence in motivated reasoning (Lodge and Taber 2013). Thereby we also move beyond existing work that studies only one dimension of core affect (Mutz 2007; Mutz and Reeves 2005; Soroka, Fournier, and Nir 2019; Soroka and McAdams 2015). Moreover, building on others who "lack[ed] direct measures of somatic response" (Lodge and Taber 2013, 208), we are the first to capture valence in response to political rhetoric using facial EMG. Applying such physiological measures we provide the first evidence for the affective underpinning of canonical political science theories.

\section{Reflections on Unexpected Findings}

There might be multiple reasons for why we rejected our hypothesis about political knowledge (H1). First, the failure to reject the null hypothesis might be due to measurement error in our short knowledge battery (Lord and Novick 2008). Second, the validity of the type of knowledge questions used by us-and others
(Brader 2006; Taber and Lodge 2006) - has been criticized (Lupia 2006; Prior 2013). Future work is therefore well advised to measure political knowledge with a longer and more diverse battery. Third, the role of knowledge as a moderator of political rhetoric is perhaps not as important as we initially thought. Close inspection of the work by Lodge and Taber (2005), for instance, shows that knowledge predicts affective links in just one of their two experiments. Finally, although higher levels of political knowledge might make affective attachments to political objects stronger, careful consideration of these political objects by those with higher knowledge could also weaken these affective attachments again.

There are four possible explanations for why we rejected our hypothesis regarding positive affect $(\mathrm{H} 4$, zygomaticus activity). First, the analyses for the zygomaticus were run on a subset of the data $(N=141)$, so the analyses are likely underpowered. Second, even though previous studies have shown that the zygomaticus is activated in response to positive stimuli (Lang et al. 1993), there is a high degree of measurement error in the zygomaticus (Larsen, Norris, and Cacioppo 2003; Tassinary, Cacioppo, and Vanman 2007). ${ }^{11}$ Third, our treatments were not particularly positive in tone. Fourth, given research on the negativity bias (Soroka, Fournier, and Nir 2019; Soroka and McAdams 2015), we should not expect a strong response from giving participants information they agree with.

We also rejected our hypothesis concerning attention (H5). Here, the problem may be that heart rate is regulated by the sympathetic and parasympathetic nervous systems and may therefore indicate both arousal and attention (Blascovich et al. 2014). One way to strictly measure attention is to analyze high-frequency heart variability only, with the respiratory sinus arrhythmia measure (Blascovich et al. 2014). Unfortunately our setup did not allow for the recording of this measure.

Our initial expectation was that issue salience should matter. However, at the individual level, issue salience consistently has zero effect. At the macro level, immigration was most salient at the time of data collection (den Ridder et al. 2016), but this is not reflected in the analyses. In fact, levels of arousal were systematically lower for the immigration issue in protocol 1. Another expectation we had was that there would be more arousal for easy issues than for hard issues. Easy issues, such as immigration and European integration, are highly symbolic, and they have been on the agenda for a long time (Carmines and Stimson 1980). Hard issues, such as climate change and inequality, are more complex issues "for which symbolic referents are not readily available" (Johnston and Wronski 2015, 3). By consequence, one could expect that "easy" issues evoke more arousal than "hard" issues. We find some evidence for this pattern (stronger arousal in EU and immigration protocol 2; see Figure 3) and some evidence against this pattern (less arousal in immigration

\footnotetext{
${ }^{11}$ Note that other studies using similar equipment in our university's lab did validly capture zygomaticus activity (Nohlen et al. 2016; Rotteveel et al. 2001).
} 
protocol 1). We welcome well-powered studies that develop and test ideas about the extent to which issue characteristics condition the affective responses of citizens.

\section{Next Steps in This Area of Research}

Theories in political science-such as motivated reasoning (Lodge and Taber 2013) and receive-accept-sample (Zaller 1992) - have theorized differently about affect. In Zaller (1992) affect is mostly cognitive evaluation, while in Lodge and Taber (2013) it is mostly unconscious. Our suggestion is to approach affect as both conscious and unconscious (see, Keltner and Gross 1999). The introduction of the circumplex model and the physiological measures of core affect capturing both arousal and valence allowed us to theorize and test the unconscious role of affect in political information processing. Going forward, a core question is how core affect relates to selfreported discrete emotions. The latter is used in the current state-of-the-art (Aarøe 2011; Delton, Petersen, and Robertson. 2018; Huddy, Mason, and Aarøe 2015; Klar 2013; Marcus, Neuman, and MacKeun 2000), and typically the two components are seen as competitors: mutually exclusive approaches to pin down the emotions of the people we study. In defending the choice for one, the other needs to be discredited. For example, McDermott $(2007,384)$ summarizes, "clearly such self-reports are not as reliable ... as the emotional physiological measures that some neuroscientists use in assessing online emotional processing such as skin conductance, heart rate and other biological measures." Similarly, physiological measures are criticized for being invalid, as they typically-and also in this study-do not correlate with self-reported emotions (Osmundsen et al. Forthcoming).

We do not share these criticisms. Keltner and Gross $(1999,468)$ explained that emotions have affective and cognitive components. Therefore, physiological and self-reported measures are different but valid indicators of emotions. These different components are dissimilar, and they are not necessarily aligned (Barrett and Satpute 2019; Evers et al. 2014). Recent work in neuroscience, for instance, suggests that different brain systems are responsible for physiological fear responses and cognitive fear responses (LeDoux and Pine 2016). In line with this, we find evidence that arousal and negative affect predict attitude change, even when controlling for self-reported emotions (see Figure 5). We interpret these findings not as evidence for the superiority of physiological measurement but rather as evidence for the different effects it has compared with self-reported emotions.

It may be particularly relevant when the affective and cognitive components of emotions align or dealign. Dealignment may be due to a lack of reflection or limited cognitive access (Lapate et al. 2014). Therefore, individual differences in "emotional granularity" (Barrett 2004) may be an important moderator. People who are more able to characterize emotions in terms of specific discrete emotions - that is, score higher on emotional granularity - might show stronger alignment between the discrete emotion they report and their physiological response compared with those who describe their emotions in "broad, global terms" - that is, score lower on emotional granularity (Barrett 2004, 37). Partisanship may also condition the alignment or dealignment of physiological affect and self-reported emotions in at least two possible ways. First, partisan parrots might echo the sentiments they hear from elites and report strong self-reported emotions, without actually having any affective (unconscious) response (Bakker, Schumacher, and Homan Forthcoming). Second, partisan ostriches have physiological responses but do not report strong self-reported emotions because they are motivated to unconsciously or consciously down-regulate their cognitive response (Bakker, Schumacher and Homan Forthcoming; Butler, Gross, and Barnard 2014). When affective and cognitive responses align or dealign they may amplify or hamper the effect of political rhetoric on political attitudes and behaviors. To conclude, we expect that studying alignment and dealignment and its consequences is a promising avenue for future research.

Within the context of further situating the circumplex model of affect in political science we see several other fruitful avenues of further study. For example, our findings speak to an emerging literature on the negativity bias in political communication (Soroka, Fournier, and Nir 2019; Soroka and McAdams 2015). The fact that we can explain variation in negative affect-but not positive affect-could be seen as an indication of the prevalence of negative over positive information. Specifically, future work could explore whether negative campaign ads (Brader 2005), negative news (Soroka, Fournier, and Nir 2019), and elite incivility (Mutz and Reeves 2005) elicit negative affect. At the same time, we also see possibilities to use the circumplex model of affect to theorize and test the role of positive affect in explaining voter turnout and campaign involvement (Brader 2005; Valentino et al. 2011). Another option is to examine other individual differences in physiological reactions to political rhetoric (Brader 2006). Our data show, for instance, that women respond with less physiological arousal in response to political rhetoric than men do. This exploratory finding contributes to a small body of research that explores if and to what extent individual differences - such as gender and ideology-can explain differences in physiological responses (Peterson et al. 2018; Soroka et al. 2016).

Going forward, we recommend that researchers consider effect sizes and the validity of physiological measures. We have benchmarked our results against, among other things, physiological responses to validated negative and positive images from the International Affective Picture System (IAPS; Arceneaux, Dunaway, and Soroka 2018; Bakker et al. 2020; Lang et al. 1993) reported elsewhere. We encourage researchers to assess the validity of the physiological measures by presenting stimuli that have been shown to evoke activity on the physiological measure of interest, such as the IAPS pictures (Lang et al. 1993). This has two advantages: (1) researchers can assess the validity of the physiological measure and (2) researchers can use the effect sizes of the validated images to interpret the effect size of the politically relevant stimulus. This 
allows researchers to directly assess whether affective responses to politics are comparable, smaller, or even larger than affective responses to other stimuli-for an illustration of this approach, see Bakker, Schumacher and Homan (Forthcoming).

To conclude, after a number of surprising political upheavals, societal and academic attention for the role of emotion in politics has rebounded. Our paper addresses this by setting the agenda for a new perspective on how emotions influence politics.

\section{SUPPLEMENTARY MATERIALS}

To view supplementary material for this article, please visit http://dx.doi.org/10.1017/S0003055420000519.

Replication materials can be found on Dataverse at: https://doi.org/10.7910/DVN/LCWR7G.

\section{REFERENCES}

Aarøe, Lene. 2011. Investigating Frame Strength: The Case of Episodic and Thematic Frames. Political Communication 28 (2): 207-26.

Abelson, Robert P. 1963. "Computer Simulation of 'Hot Cognition.", In Computer Simulation of Personality, eds. Silvan S. Tomkins, and Samuel Messick, 277-98. New York: Wiley.

Arceneaux, Kevin, Johanna Dunaway, and Stuart N. Soroka. 2018. "Elites Are People, Too: The Effects of Threat Sensitivity on Policymakers Spending Priorities.” Plos ONE 13 (4): E0193781.

Bakker, Bert N., Gijs Schumacher, Claire Gothreau, and Kevin Arceneaux. 2020. "Conservatives and Liberals Have Similar Physiological Responses to Threats: Evidence from Three Replications." Nature Human Behaviour 4 (6): 613-21.

Bakker, Bert N., Gijs Schumacher, and Maaike D. Homan. Forthcoming. "Yikes! Are We Disgusted By Politicians?" Politics and the Life Sciences.

Barrett, Lisa Feldman. 2004. "Feelings or Words? Understanding the Content in Self-Report Ratings of Experienced Emotion." Journal of Personality and Social Psychology 87 (2): 266-81.

Barrett, Lisa Feldman, and Ajay B. Satpute. 2019. "Historical Pitfalls and New Directions in the Neuroscience of Emotion." Neuroscience Letters 69: 9-18.

Blascovich, Jim, Eric Vanman, Wendy Mendes, and Sally Dickerson. 2014. Social Psychophysiology for Social and Personality Psychology. London: Sage Publications.

Brader, Ted. 2005. "Striking a Responsive Chord: How Political Ads Motivate and Persuade Voters by Appealing to Emotions." American Journal of Political Science 49 (2): 388-405.

Brader, Ted. 2006. Campaigning for Hearts and Minds: How Emotional Appeals in Political Ads Work. Chicago: University Of Chicago Press.

Bradley, Margaret M., Maurizio Codispoti, Bruce N. Cuthbert, and Peter J Lang. 2001. "Emotion and Motivation I: Defensive and Appetitive Reactions in Picture Processing." Emotion 1 (3): 276-98.

Butler, Emily A., James J. Gross, and Kobus Barnard. 2014. "Testing the Effects of Suppression and Reappraisal on Emotional Concordance Using a Multivariate Multilevel Model.” Biological Psychology 9: 6-18.

Cacioppo, John T., Richard E. Petty, Mary E. Losch, and Hai Sook Kim. 1986. "Electromyographic Activity over Facial Muscle Regions Can Differentiate the Valence and Intensity of Affective Reactions." Journal of Personality and Social Psychology 50 (2): 260-8.

Camerer, Colin F., Anna Dreber, Felix Holzmeister, Teck-Hua Ho, Jürgen Huber, Magnus Johannesson, Michael Kirchler, et al. 2018. "Evaluating the Replicability of Social Science Experiments in Nature and Science between 2010 and 2015." Nature Human Behaviour 2 (9): 637-44.
Carmines, Edward G., and James A. Stimson. 1980. "The Two Faces of Issue Voting." American Political Science Review 74 (1): 78-91.

Claassen, Christopher. 2020. "Does Public Support Help Democracy Survive?" American Journal of Political Science 64 (1): 118-34.

Daignault, Pénélope, Stuart N. Soroka, and Thierry Giasson. 2013. "The Perception of Political Advertising during an Election Campaign: A Measure of Cognitive and Emotional Effects." Canadian Journal of Communication 38 (2): 167-86.

Dawson, Michael E., Anne M. Schell, and Diane L. Filion. 2007.

"The Electrodermal System." In Handbook of Psychophysiology, eds. John T. Cacioppo, Louis G. Tassinary, and Gary G. Berntson, 159-81. Cambridge: Cambridge University Press.

Delton, Andrew W., Michael Bang Petersen, and Theresa E. Robertson. 2018. "Partisan Goals, Emotions, and Political Mobilization: The Role of Motivated Reasoning in Pressuring Others to Vote." The Journal of Politics 80 (3): 890-902.

Den Ridder, J., P. Dekker, P. Van Houwelingen, and E. Schrijver. 2016. Burgerperspectieven 2016/1. Den Haag: Sociaal en Cultureel Planbureau.

Erisen, Cengiz, Milton Lodge, and Charles S. Taber. 2014. "Affective Contagion in Effortful Political Thinking." Political Psychology 35 (2): 187-206.

Evers, Catharine, Henrik Hopp, James J. Gross, Agneta H. Fischer, Antony S. R. Manstead, and Iris B. Mauss. 2014. "Emotion Response Coherence: A Dual-Process Perspective.” Biological Psychology 9: 43-9.

Gazendam, Femke J., Jan H. Kamphuis, and Merel Kindt. 2013. "Deficient Safety Learning Characterizes High Trait Anxious Individuals." Biological Psychology 92 (2): 342-52.

Hietanen, Jari K., Veikko Surakka, and Ilkka Linnankoski. 1998. "Facial Electromyographic Responses to Vocal Affect Expressions." Psychophysiology 35 (5): 530-6.

Huddy, Leonie, Lilliana Mason, and Lene Aarøe. 2015. "Expressive Partisanship: Campaign Involvement, Political Emotion, and Partisan Identity." American Political Science Review 109 (1): 1-17.

Imai, Kosuke, Luke Keele, and Dustin Tingley. 2010. “A General Approach to Causal Mediation Analysis." Psychological Methods 15 (4): 309.

Johnston, Christopher D., and Julie Wronski. 2015. "Personality Dispositions and Political Preferences across Hard and Easy Issues." Political Psychology 36 (1): 35-53.

Kalla, Joshua L., and David E. Broockman. 2020. "Reducing Exclusionary Attitudes through Interpersonal Conversation: Evidence from Three Field Experiments." American Political Science Review 114 (2): 410-25.

Kaplan, Jonas T., Sarah I. Gimbel, and Sam Harris. 2016. "Neural Correlates of Maintaining One's Political Beliefs in the Face of Counterevidence." Scientific Reports 6: 39589.

Keltner, Dacher, and James J. Gross. 1999. "Functional Accounts of Emotions." Cognition \& Emotion 13 (5): 467-80.

Klar, Samara. 2013. "The Influence of Competing Identity Primes on Political Preferences." The Journal of Politics 75 (4): 1108-24.

Lang, Annie, John Newhagen, and Byron Reeves. 1996. "Negative Video as Structure: Emotion, Attention, Capacity, and Memory." Journal of Broadcasting \& Electronic Media 40 (4): 460-77.

Lang, Peter J., Mark K. Greenwald, Margaret M. Bradley, and Alfons O. Hamm. 1993. "Looking at Pictures: Affective, Facial, Visceral, and Behavioral Reactions.” Psychophysiology 30 (3): 261-73.

Lapate, Regina C., Bas Rokers, Tianyi Li, and Richard J. Davidson. 2014. "Nonconscious Emotional Activation Colors First Impressions: A Regulatory Role for Conscious Awareness." Psychological Science 25 (2): 349-57.

Larsen, Jeff T., Catherine J. Norris, and John T. Cacioppo. 2003. "Effects of Positive and Negative Affect on Electromyographic Activity over Zygomaticus Major and Corrugator Supercilii." Psychophysiology 40 (5): 776-85.

Lau, Richard R. 1989. Construct Accessibility and Electoral Choice. Political Behavior 11 (1): 5-32.

Ledoux, Joseph E., and Daniel S. Pine. 2016. Using Neuroscience to Help Understand Fear and Anxiety: A Two-System Framework." American Journal of Psychiatry 173 (11): 1083-93.

Lodge, Milton, and Charles S. Taber. 2005. "The Automaticity of Affect for Political Leaders, Groups, and Issues: An Experimental 
Test of the Hot Cognition Hypothesis.” Political Psychology 26 (3): 455-82.

Lodge, Milton, and Charles S. Taber. 2013. The Rationalizing Voter. Cambridge: Cambridge University Press.

Lord, Frederic M., and Melvin R. Novick. 2008. Statistical Theories of Mental Test Scores. Charlotte, NC: Information Age Publishing.

Lupia, Arthur. 2006. "How Elitism Undermines the Study of Voter Competence." Critical Review 18 (1-3): 217-32.

Marcus, George E., W. Russell Neuman, and Michael Mackuen. 2000. Affective Intelligence and Political Judgment. Chicago: University of Chicago Press.

Mcdermott, Rose. 2007. "Cognitive Neuroscience and Politics: Next Steps." In The Affect Effect: Dynamics of Emotion in Political Thinking and Behavior, eds. W. Russell Neuman, George E. Marcus, Ann N. Crigler, and Michael Mackuan, 375-97. Cambridge: Cambridge University Press.

Morris, James P., Nancy K. Squires, Charles S. Taber, and Milton Lodge. 2003. "Activation of Political Attitudes: A

Psychophysiological Examination of the Hot Cognition Hypothesis." Political Psychology 24 (4): 727-45.

Mutz, Diana C. 2007. "Effects of 'In-Your-Face' Television Discourse on Perceptions of a Legitimate Opposition." American Political Science Review 101 (4): 621-35.

Mutz, Diana C., and Byron Reeves. 2005. "The New Videomalaise: Effects of Televised Incivility on Political Trust." American Political Science Review 99 (1): 1-15.

Nohlen, Hannah U., Frenk Van Harreveld, Mark Rotteveel, Ard J. Barends, and Jeff T. Larsen. 2016. Affective Responses to Ambivalence Are Context-Dependent: A Facial EMG Study on the Role of Inconsistency and Evaluative Context in Shaping Affective Responses to Ambivalence." Journal of Experimental Social Psychology 65: 42-51.

Osmundsen, Mathias, David Hendry, Lasse Laustsen, Kevin Smith, and Michael Bang Petersen. Forthcoming. "The Psychophysiology of Political Ideology: Replications, Reanalysis and Recommendations." The Journal of Politics.

Oxley, Douglas R., Kevin B. Smith, John R. Alford, Matthew V. Hibbing, Jennifer L. Miller, Mario Scalora, Peter K. Hatemi, and John R. Hibbing. 2008. "Political Attitudes Vary with Physiological Traits." Science 321 (5896): 1667-70.

Petersen, Michael Bang, Ann Giessing, and Jesper Nielsen. 2015. "Physiological Responses and Partisan Bias: Beyond Self-Reported Measures of Party Identification.” Plos One 10 (5): E0126922.

Peterson, Johnathan Caleb, Carly Jacobs, John Hibbing, and Kevin Smith. 2018. "In Your Face: Emotional Expressivity as a Predictor of Ideology." Politics and the Life Sciences 37 (1): 53-67.

Potter, Robert F., and Paul Bolls. 2012. Psychophysiological Measurement and Meaning: Cognitive and Emotional Processing of Media. New York: Routledge.

Potter, Robert F., and Justin Robert Keene. 2012. "The Effect of Sports Fan Identification on the Cognitive Processing of Sports News." International Journal of Sport Communication 5 (3): 348-67.

Prior, Markus. 2013. "Visual Political Knowledge: A Different Road to Competence?" The Journal of Politics 76 (1): 41-57.

Rabinowitz, George, and Stuart Elaine Macdonald. 1989. “A Directional Theory of Issue Voting." American Political Science Review 83 (1): 93-121.

Renshon, Jonathan, Jooa Julia Lee, and Dustin Tingley. 2015. "Physiological Arousal and Political Beliefs." Political Psychology 36 (5): 569-85.
Rotteveel, Mark, Paulien De Groot, Arjen Geutskens, and R. Hans Phaf. 2001. "Stronger Suboptimal Than Optimal Affective Priming?" Emotion 1 (4): 348.

Russell, James A. 1980. “A Circumplex Model of Affect.” Journal of Personality and Social Psychology 39 (6): 1161-78.

Sevenster, Dieuwke, Tom Beckers, and Merel Kindt. 2012.

"Retrieval per se Is Not Sufficient to Trigger Reconsolidation of Human Fear Memory." Neurobiology of Learning and Memory 97 (3): $338-45$

Shepherd, Michael E., and Hye Young You. 2020. "Exit Strategy: Career Concerns and Revolving Doors in Congress." American Political Science Review 114 (1): 270-84.

Soroka, Stuart N. 2014. Negativity in Democratic Politics: Causes and Consequences. Cambridge: Cambridge University Press.

Soroka, Stuart N., Patrick Fournier, and Lilach Nir. 2019. "CrossNational Evidence of a Negativity Bias in Psychophysiological Reactions to News." Proceedings of the National Academy of Sciences 116 (38): 18888-92.

Soroka, Stuart N., Elisabeth Gidengil, Patrick Fournier, and Lilach Nir. 2016. "Do Women and Men Respond Differently to Negative News?" Politics \& Gender 12 (2): 344-68.

Soroka, Stuart N., and Stephen Mcadams. 2015. "News, Politics, and Negativity." Political Communication 32 (1): 1-22.

Suhay, Elizabeth, and Cengiz Erisen. 2018. "The Role of Anger in the Biased Assimilation of Political Information." Political Psychology 39 (4): 793-810.

Taber, Charles S., and Milton Lodge. 2006. "Motivated Skepticism in the Evaluation of Political Beliefs." American Journal of Political Science 50 (3): 755-69.

Tassinary, Louis G., John Cacioppo, and Eric Vanman. 2007. "The Skeletomotor System: System Electromyography." In Handbook of Psychophysiology, eds. John T. Cacioppo, Louis G. Tassinary, and Gary G. Berntson, 267-99. Cambridge: Cambridge University Press.

Valentino, Nicholas A., Ted Brader, Eric W. Groenendyk, Krysha Gregorowicz, and Vincent L. Hutchings. 2011. "Election Night's Alright for Fighting: The Role of Emotions in Political Participation." The Journal of Politics 73 (01): 156-70.

Van Oyen Witvliet, Charlotte, and Scott R. Vrana. 1995. "Psychophysiological Responses as Indices of Affective Dimensions." Psychophysiology 32 (5): 436-43.

Venables, P. H., and D. A. Mitchell. 1996. "The Effects of Age, Sex and Time of Testing on Skin Conductance Activity." Biological Psychology 43 (2): 87-101.

Wagner, Michael W., Kristen D. Deppe, Carly M. Jacobs, Amanda Friesen, Kevin B. Smith, and John R. Hibbing. 2014. "Beyond Survey Self-Reports: Using Physiology to Tap Political Orientations." International Journal of Public Opinion Research 27 (3): 303-17.

Wang, Zheng, Alyssa C. Morey, and Jatin Srivastava. 2014. Motivated Selective Attention during Political Ad Processing: The Dynamic Interplay between Emotional Ad Content and Candidate Evaluation." Communication Research 41 (1): 119-56.

Wexler, Bruce E., Stephen Warrenburg, Gary E. Schwartz, and Larry D. Janer. 1992. "EEG and EMG Responses to EmotionEvoking Stimuli Processed without Conscious Awareness." Neuropsychologia 30 (12): 1065-79.

Zaller, John R. 1992. The Nature and Origins of Mass Opinion. Cambridge: Cambridge University Press. 